\title{
Transitional care services: a quality and safety process improvement program in neurosurgery
}

\author{
${ }^{*}$ Faith C. Robertson, BS, ${ }^{1,2}$ Jessica L. Logsdon, PA-C, MHA, ${ }^{2,3}$ \\ Hormuzdiyar H. Dasenbrock, MD, MPH, ${ }^{1-3}$ Sandra C. Yan, BS, BA, ${ }^{2,3}$ \\ Siobhan M. Raftery, RN, BSN, CNRN, ${ }^{2,3}$ Timothy R. Smith, MD, PhD, MPH, ${ }^{1-3}$ \\ and William B. Gormley, MD, MPH, MBA ${ }^{1-3}$
}

${ }^{1}$ Harvard Medical School; and ${ }^{2}$ Cushing Neurosurgical Outcomes Center, ${ }^{3}$ Department of Neurological Surgery, Brigham and
Women's Hospital, Boston, Massachusetts

OBJECTIVE Readmissions increasingly serve as a metric of hospital performance, inviting quality improvement initiatives in both medicine and surgery. However, few readmission reduction programs have targeted surgical patient populations. The objective of this study was to establish a transitional care program (TCP) with the goal of decreasing length of stay (LOS), improving discharge efficiency, and reducing readmissions of neurosurgical patients by optimizing patient education and postdischarge surveillance.

METHODS Patients undergoing elective cranial or spinal neurosurgery performed by one of 5 participating surgeons at a quaternary care hospital were enrolled into a multifaceted intervention. A preadmission overview and establishment of an anticipated discharge date were both intended to set patient expectations for a shorter hospitalization. At discharge, in-hospital prescription filling was provided to facilitate medication compliance. Extended discharge appointments with a neurosurgery TCP-trained nurse emphasized postoperative activity, medications, incisional care, nutrition, signs that merit return to medical attention, and follow-up appointments. Finally, patients received a surveillance phone call 48 hours after discharge. Eligible patients omitted due to staff limitations were selected as controls. Patients were matched by sex, age, and operation type-key confounding variables-with control patients, who were eligible patients treated at the same time period but not enrolled in the TCP due to staff limitation. Multivariable logistic regression evaluated the association of TCP enrollment with discharge time and readmission, and linear regression with LOS. Covariates included matching criteria and Charlson Comorbidity Index scores.

RESULTS Between 2013 and 2015, 416 patients were enrolled in the program and matched to a control. The median patient age was 55 years (interquartile range $44.5-65$ years); $58.4 \%$ were male. The majority of enrolled patients underwent spine surgery (59.4\%, compared with $40.6 \%$ undergoing cranial surgery). Hospitalizations averaged 62.1 hours for TCP patients versus 79.6 hours for controls (a $16.40 \%$ reduction, $95 \% \mathrm{CI} 9.30 \%-23.49 \% ; p<0.001$ ). The intervention was associated with a higher proportion of morning discharges, which was intended to free beds for afternoon admissions and improve patient flow $(\mathrm{OR} 3.13,95 \% \mathrm{Cl} 2.27-4.30 ; p<0.001)$, and decreased 30 -day readmissions $(2.5 \%$ vs 5.8\%; OR 2.43, 95\% Cl 1.14-5.27; $p=0.02$ ).

CONCLUSIONS This neurosurgical TCP was associated with a significantly shorter LOS, earlier discharge, and reduced 30-day readmission after elective neurosurgery. These results underscore the importance of patient education and surveillance after hospital discharge.

https://thejns.org/doi/abs/10.3171/2017.2.JNS161770

KEY WORDS discharge program; hospital readmissions; length of stay; quality; patient education; safety; transitional care

$\mathrm{H}$ EALTH care reform in the United States emphasizes value-based parameters to improve quality, efficiency, cost-effectiveness, and patient experience. ${ }^{36}$ Readmissions are a common metric of hospital performance, as 30-day readmissions are associated with increased mortality and cost.11,46 Annual Medicare readmissions alone amount to $\$ 17.4$ billion, leading Medicare and Accountable Care Organizations to institute financial penalties for high readmission rates. ${ }^{11,23,49}$ Hospitals exceeding particular 30-day readmission rates for pneumo-

ABBREVIATIONS $\mathrm{CCI}=$ Charlson Comorbidity Index; $\mathrm{LOS}=$ length of stay; $\mathrm{TCP}=$ transitional care program.

SUBMITTED July 6, 2016. ACCEPTED February 2, 2017.

INCLUDE WHEN CITING Published online July 14, 2017; DOI: 10.3171/2017.2.JNS161770.

* Ms. Robertson and Ms. Logsdon contributed equally to this work. 
nia, myocardial infarction, heart failure, and total hip and knee replacements are now facing reduced financial reimbursements; other surgical specialties, including neurological surgery, may incur such penalties in the future. . $^{13,24}$

However, the extent to which readmissions-particularly after elective surgery-are preventable remains controversial. ${ }^{7,37,42,48}$ Evidence suggests that readmission rates may be reduced though discharge planning strategies and targeted patient education, as inadequate patient engagement and incomplete patient comprehension affect compliance and recognition of complications, thereby increasing readmission risk. ${ }^{1,31-33}$ Institutions have also recognized the need to address inefficient discharges, prolonged hospitalization, unnecessary cost, and patient dissatisfaction. ${ }^{17,19,26,33,47}$ Consequently, recent health care programs have emphasized patient education and transitional care; examples include the Transitional Care Model, Project RED (Re-Engineered Discharge), and Project BOOST (Better Outcomes by Optimizing Safe Transitions), which have demonstrated readmission reduction in nonsurgical specialties. ${ }^{4,22,35,39,50}$ In surgical practice, however, quality improvement has focused primarily on perioperative measures, with less attention toward postdischarge management. . $^{10,16,25,29,30,44,45}$

In this study, a novel transitional care program (TCP) was implemented to improve transparency and fluidity of the postoperative process for neurosurgical patients. The intervention was multifaceted; key components included a preadmission overview, documented anticipated discharge date, daily contact with the transitional care team during the hospitalization, 24-hour advance notice of discharge, in-hospital prescription filling, a 30-minute exit consultation at discharge with a trained discharge nurse for provision of surgery-specific education, and a telephone call 48 hours after discharge. Directed patient education at discharge was intended to resolve common questions regarding medications, incision care, nutritional optimization, and signs that warrant medical attention. The multidisciplinary effort aimed to reduce length of stay (LOS) and 30-day readmissions, while enhancing the patient experience and quality of care.

\section{Methods \\ Study Site}

The neurosurgery TCP was implemented at a large American quaternary academic referral center under a parent health care organization. In the department of neurosurgery, the posthospitalization transition was identified as an ideal target for quality improvement. The TCP was launched in 2013 to improve clinical care surrounding patient discharge.

\section{Inclusion and Exclusion Criteria}

Patients were eligible for the TCP if scheduled for elective neurological surgery by any one of 5 participating surgeons and were expected to have a discharge disposition to home. Three participating neurosurgeons were spinal subspecialists, and the remaining 2 were cranial surgeons. Because patient accrual was limited by the capacity of our service, approximately half of the patients of each surgeon were included; patients were enrolled based on the estimated date of discharge, not clinical information, until the capacity of discharge appointments for a respective discharge day was met. Eligible patients who were omitted due to staff limitations were matched to TCP participants by sex, age, and operation and used as controls in analysis. Patients discharged to a facility other than home were excluded. The goal of this program was to provide patient education and surveillance for patients discharged to home; those discharged to health care settings with nursing staff to assist with wound care, medication compliance, and complication prevention could potentially dilute the intervention.

\section{Preoperative Period}

Eligible patients were contacted to enroll in the TCP. TCP participants received a program overview and anticipated discharge date, which was determined by each surgeon's expected LOS per procedure, indicating the anticipated discharge date was intended to improve patients' expectations for how long the hospital LOS would be. Electronic and postal notifications were sent to patients and their families. Information packets included surgeonand procedure-specific educational materials describing the operative procedure and standard postoperative care, anticipated LOS, and discharge appointment time. Materials also emphasized the central role of family engagement in the process, including participation in the discharge appointment and transfer to home.

\section{Postoperative Period}

Postoperatively, the patient was monitored daily by the TCP team members, who attended multidisciplinary rounds, updated discharge appointments, sent updates to referring physicians, and met with patients and families. The day before discharge, medication plans (including tapers for dexamethasone and narcotics) were written, critical prescriptions were relayed to the pharmacy for preparation, and the remaining prescriptions were placed in the patient's chart. Critical prescriptions involved those identified as causes for medication noncompliance and hospital readmission in the experience of the participating surgeons, including steroid tapers, antiepileptic drugs, pain medications, and newly prescribed antibiotics and anticoagulants. Hospital administration and pharmacy personnel participated in the program through an agreement to fund and fill these high-priority prescriptions. Finally, the plan for the patient's transportation to home was confirmed, and, if caregivers were unable to assist, the TCP staff arranged for alternative transportation and ensured that the home situation was prepared and safe for the patient.

\section{Discharge Appointment}

On the day of discharge, a TCP nurse collected the essential patient medications from the outpatient pharmacy in preparation for discharge appointments. To achieve efficient discharges, all appointments were scheduled before noon. At discharge, the patient was transported by the nurse to a dedicated education suite for a consultation 
with a neurosurgery TCP-trained nurse. The discharge suite was designed to provide a space for the outgoing patient, thereby opening the hospital bed for new admissions or transfers from the intensive care unit. Early discharges are particularly critical for surgical services to optimize patient flow from the operating room to the intensive care unit or the perianesthesia care unit, and such improvements in efficiency enhance bed availability for the hospital. Each appointment was scheduled for 30 minutes and involved education tailored to both procedure and patient. The following topics were addressed: activity, medications (safety, dosing, and administration), incisional care, nutrition, and follow-up appointments. The patient was given a discharge folder with all critical medications, including prefilled prescriptions. Direct medication procurement and review was intended to increase patient compliance.

\section{Posthospitalization Follow-Up}

A follow-up telephone call was made within 48 hours of discharge to assess pain, medication compliance, anticoagulant or antithrombotic therapy, incisional care, activity, and any remaining questions. Follow-up appointments (scheduled at the discretion of the primary surgeon but often 2 weeks postoperatively) were confirmed. Outstanding issues were addressed by the discharge nurse and, if necessary, relayed to the surgeon. A primary contact was designated to facilitate any remaining questions.

In summary, the fundamentals of the TCP program differed from the traditional discharge process by the following: prior discharges lacked preoperative expectations for anticipated discharge, critical medications were filled by the patient after discharge, dedicated educational exit interviews with mandatory caregiver co-attendance were absent, and there was a lack of postdischarge surveillance with telephone follow-up. Additionally, discharge education was historically provided by routine floor nurses who did not necessarily have dedicated training in discharge education; therefore, the TCP provided a level of standardization in patient education at discharge, in addition to focusing on content discussed.

\section{Survey}

An optional, electronic survey was available to participants after program completion via an email link. Additionally, a small subset of non-TCP participants was recruited to complete the survey. Respondents were asked to report satisfaction during hospitalization and discharge.

\section{Outcomes Assessment}

Outcome measures were predetermined, and data were collected through intake forms, longitudinal data collection, hospital medical records, and billing statements. Primary outcome measures were 30-day unplanned hospital readmissions (to the same institution as the index operation) and hospital LOS, the latter of which was evaluated continuously by the number of hours of the initial hospitalization. Secondary analysis included time of discharge (with an early discharge defined as before 12:00 PM). Morning discharge was emphasized as part of an institu- tionally guided effort to facilitate patient progression. A Charlson Comorbidity Index (CCI) score was calculated for each patient using recorded secondary diagnoses. ${ }^{9}$

\section{Statistical Analysis}

Statistical analyses were performed using STATA (version 13.0, StataCorp). Controls were chosen using automated selection (STATA supplemental program CCMATCH) from the aforementioned elective-case patients who did not receive TCP services due to staff limitations. Patients were matched by sex, age, and operation to reduce confounding from these variables. As the number of patients in the control group exceeded that of the intervention group, all 416 TCP participants were control matched according to the aforementioned variables. Descriptive statistics were performed. Outcomes were evaluated with multivariable regression after including matching criteria (age, sex, and operation) and CCI score as covariates to prevent selection bias. Logistic regression was used for dichotomous outcomes; continuous outcomes (e.g., LOS) were evaluated with linear regression, and data were logarithmically transformed prior to analysis due to nonnormal distribution. Concordance (C) statistics and $\mathrm{R}^{2}$ assessed the discriminatory capacity of logistic and linear regression models, respectively. A sensitivity analysis was performed to evaluate if cranial or spinal patients sustained differential results from the program: in this analysis, regression constructs included an interaction term between operation type (dichotomized as cranial vs spinal) and the TCP. Probability values $<0.05$ were considered significant.

\section{Results \\ Demographics}

The TCP, outlined in Fig. 1, enrolled 416 patients between October 2013 and June 2015. As there were more control patients than TCP enrollees, all 416 patients were able to be matched by age, sex, and operation. The median patient age was 55 years (interquartile range 44.5-65 years), and spinal surgeries were more prevalent than cranial, making up $59.4 \%$ of program participants. Additional patient characteristics are described in Table 1.

\section{Outcomes}

Enrollment in the program was associated with significantly lower odds of an unplanned hospital readmission (2.5\% vs 5.8\%; OR 2.43, 95\% CI 1.14-5.27; $\mathrm{p}=0.02$ ). Among patients who required readmission, the time from discharge to readmission did not differ between controls and TCP participants $(\mathrm{p}=0.75)$. The TCP also resulted in shorter LOS, as hospitalizations averaged 62.1 hours (SD 35.4 hours) for TCP patients compared with 79.6 hours (SD 59.8 hours) for controls (16.40\% decrease in stay, $95 \%$ CI 9.30\%-23.49\%; p < 0.001) (Table 2 and Fig. 2). Discharging patients by noon was intended to increase bed availability for afternoon admissions, and only $18.0 \%$ of controls, compared with $40.4 \%$ of TCP patients, were discharged before $12: 00$ PM (OR $0.31,95 \%$ CI $0.23-0.43$; $p$ $<0.001)$.

A sensitivity analysis evaluated for the potential interaction between enrollment in the TCP and operation type 


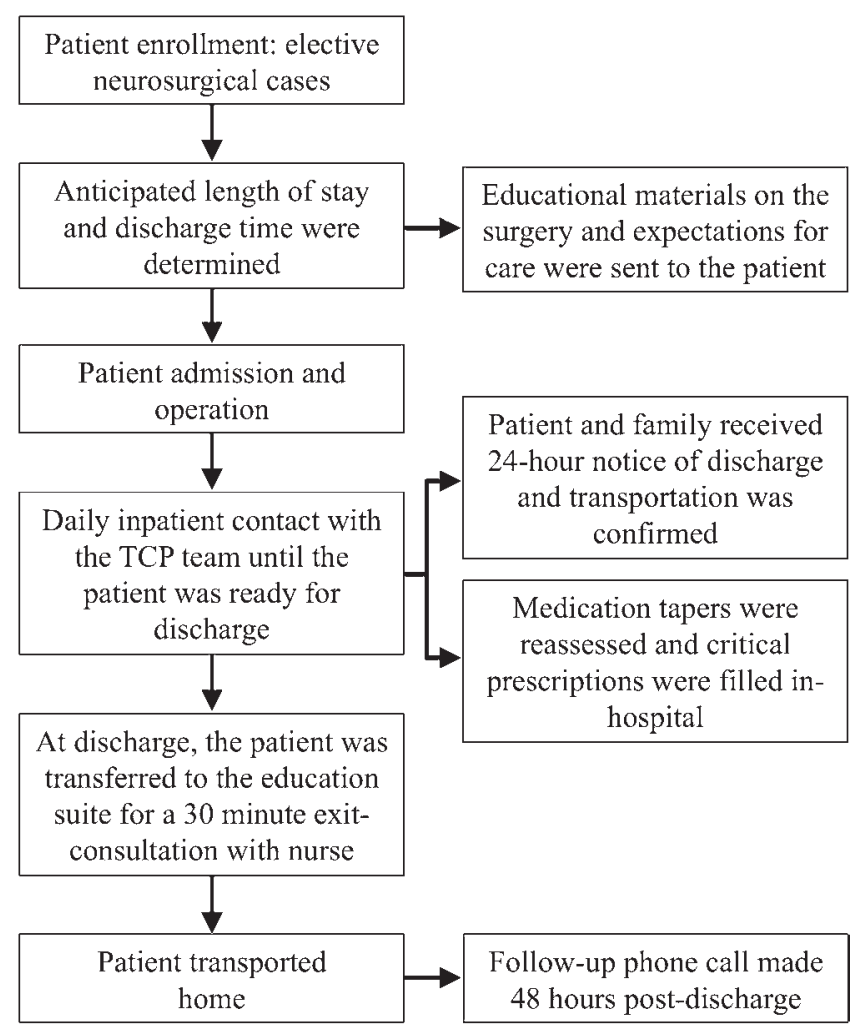

FIG. 1. Flow diagram depicting the components of the TCP.

(dichotomized for the sensitivity analysis as cranial vs spinal). However, cranial versus spinal surgery was not found to be a statistically significant effect measure modifier of the benefit of the TCP on any outcomes evaluated (data not shown). This suggests that the utility of the TCP extends to both cranial and spinal patients postoperatively.

\section{Patient Satisfaction}

Finally, results of postdischarge surveys (Supplemental Table 1) demonstrated a positive reflection on the new discharge process. One hundred TCP enrollees and 15 controls completed the survey. There was a significant difference between the 2 groups for all survey questions. Before surgery, $72.2 \%$ of TCP patients agreed or strongly agreed to having an understanding of the discharge process, compared with $69.2 \%$ of control patients $(\mathrm{p}=0.04)$. A similar pattern was elicited for patients' understanding of their medical condition. Greater than $90 \%$ of TCP patients felt comfortable with postoperative care and follow-up plans. While only $60 \%$ of control patients reported that our traditional discharge service was helpful and recommendable, over $90 \%$ of TCP patients reflected positively on the pro$\operatorname{gram}(\mathrm{p}<0.001)$.

\section{Discussion}

Health care quality metrics, including hospital LOS, efficiency of discharge, and readmission rates, are gaining significance as benchmarks for hospital performance. . $^{13,24}$ Many hospitals have implemented protocols to enhance health care delivery surrounding patient discharge, and,
TABLE 1. The demographics of the transitional care population and their respective controls, who were matched by sex, age, and operation

\begin{tabular}{|c|c|c|c|}
\hline Variable & $\begin{array}{c}\text { Total } \\
(\mathrm{n}=832)\end{array}$ & $\begin{array}{l}\text { Controls } \\
(n=416)\end{array}$ & $\begin{array}{c}\text { TCP } \\
(n=416)\end{array}$ \\
\hline Mean age in yrs (SD) & $54.0(14.3)$ & $54.0(14.1)$ & $54.0(14.4)$ \\
\hline \multicolumn{4}{|l|}{ Sex $(\%)$} \\
\hline Male & 58.4 & 58.4 & 58.4 \\
\hline Female & 41.6 & 41.6 & 41.6 \\
\hline \multicolumn{4}{|l|}{ Operation (\%) } \\
\hline Ventricular shunt placement & 2.2 & 2.2 & 2.2 \\
\hline Craniotomy for tumor & 24.5 & 24.5 & 24.5 \\
\hline $\begin{array}{l}\text { Anterior cervical decompres- } \\
\text { sion \& fusion }\end{array}$ & 15.1 & 15.1 & 15.1 \\
\hline $\begin{array}{l}\text { Posterior cervical decom- } \\
\text { pression w/ or w/o fusion }\end{array}$ & 5.1 & 5.1 & 5.1 \\
\hline Lumbar spine decompression & 13.7 & 13.7 & 13.7 \\
\hline Lumbar spine fusion & 12.5 & 12.5 & 12.5 \\
\hline Transsphenoidal surgery & 1.4 & 1.4 & 1.4 \\
\hline Other cranial surgery* & 12.5 & 12.5 & 12.5 \\
\hline Other spinal surgery $\dagger$ & 13.0 & 13.0 & 13.0 \\
\hline \multicolumn{4}{|l|}{$\mathrm{CCl}(\%)$} \\
\hline 0 & 59.5 & 57.0 & 62.0 \\
\hline 1 & 11.3 & 9.6 & 13.0 \\
\hline$\geq 2$ & 29.2 & 33.4 & 25.0 \\
\hline
\end{tabular}

* Included procedures were trigeminal root decompression, partial pinealectomy, skull biopsy, and other craniotomy.

$\dagger$ Included resection of spinal tumors or other exploration and decompression of the spinal canal.

while the majority have focused on medical patients (Project RED, Project BOOST), ${ }^{8,22}$ few are targeting surgical patient discharge planning. Elective cases with high readmission rates are a highly desirable intervention target. Coronary artery bypass graft surgery was one of the first surgical initiatives. The STAAR (State Action on Avoidable Rehospitalizations) initiative focused on patient education and follow-up appointments and decreased 30-day readmission rates from $25.8 \%$ for controls to $12.0 \%$ for STAAR participants. ${ }^{3}$ Neurosurgery is another field that could greatly benefit from readmission reduction interventions; the reported 30-day readmission rates have primarily ranged between $10 \%$ and $15 \%$, with variations by operation between $4.4 \%$ after elective spine surgery and 23.9\% after cerebrovascular intervention., $5,634,37,46,48$ Many neurosurgical studies have revealed risk factors and predictors for readmission, such as age, number of comorbidities, and socioeconomic status, but the extent to which readmissions are preventable remains debated..$^{30,37,42,48}$

While predictors of readmission are well described, few neurosurgical groups have published methods of using such data to inform readmission reduction programs. In Finland, implementation of the WHO's surgical safety checklist reduced unplanned neurosurgical readmissions from $25 \%$ to $10 \%$; preventing wound complications was the primary determinant. ${ }^{30}$ An endoscopic neurosurgery group in Texas recently implemented a short-stay protocol 
TABLE 2. TCP patient and control outcomes*

\begin{tabular}{|c|c|c|c|c|c|c|}
\hline Variable & Controls $(n=416)$ & $\operatorname{TCP}(n=416)$ & Coefficient ( $\beta$ ) or OR† & $95 \% \mathrm{Cl}$ & p Value & $\mathrm{C}$ or $\mathrm{R}^{2}$ \\
\hline Mean LOS in hrs (SD) & $79.6(59.8)$ & $62.1(35.4)$ & $\beta: 16.40 \%$ & $9.30-23.49 \%$ & $<0.001 \ddagger$ & $R^{2}: 0.21$ \\
\hline Discharge by noon (\%) & 18.0 & 40.4 & OR: 0.31 & $0.23-0.43$ & $<0.001 \ddagger$ & C: 0.67 \\
\hline Readmission (\%) & 5.8 & 2.5 & OR: 2.43 & $1.14-5.27$ & $0.02 \ddagger$ & C: 0.74 \\
\hline
\end{tabular}

to reduce hospital LOS after pituitary adenoma surgery, and included follow-up phone calls to sustain a low rate of complications and readmissions. ${ }^{45}$ The initiation of neurosurgical interventions is encouraging, and more research is needed to optimize treatment protocols surrounding patient discharge and readmissions in neurosurgery. To our knowledge, our study is the first to report a comprehensive care program designed to reduce LOS and readmissions within neurosurgery.

The TCP was designed and implemented within the department of neurosurgery at an academic referral center with an intention to improve 3 value-based health metrics: LOS, timing of hospital discharge, and unplanned 30-day readmissions. All 3 end points affect the quality of care and hospital costs. Both pre- and postoperative education was implemented to facilitate an informed hospital discharge. The intervention included a preadmission overview of the TCP and anticipated discharge date, postoperative daily contact with the transitional care team, 24-hour advance notice of discharge to the care team, in-hospital prescription filling, a 30-minute exit consultation with nurses trained in discharge education, and a follow-up telephone call 48 hours postdischarge. Overall, the TCP assisted in improving patient and caregiver expectations, reducing LOS, vacating beds for new admissions, and reducing hospital readmissions.

Previously, the discharge process at our hospital lacked specific guidelines for discharge times. This contributed to a higher likelihood that patients would occupy beds while awaiting the completion of rounds, documentation of discharge, and organization of patient transport home, all of which had the potential to delay admissions

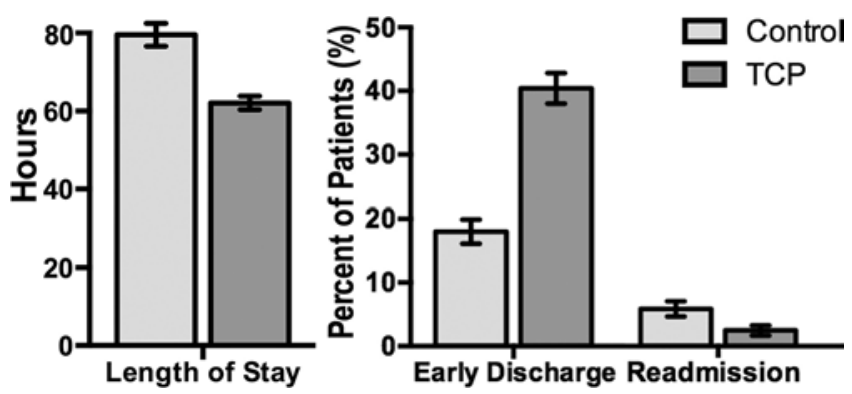

FIG. 2. Postoperative outcomes stratified by enrollment in the TCP. The TCP included preoperative education and discharge date assignment, daily postoperative visits from the TCP team during rounds, essential prescriptions filled in-hospital, one-on-one exit consultation, and a follow-up phone call 48 hours after discharge. Bars represent the mean and error bars the SD. of new patients. The TCP led to more efficient discharges; patients enrolled in the program had increased odds of morning discharge, which facilitated patient flow and a significantly shorter hospitalization. Fundamentally, the program engaged health practitioners and family members in the discharge scheduling and set clear expectations for hospital departure. Having a predetermined discharge appointment influenced patients' expectations about LOS and timing of discharge. Recent data suggest that patient expectations predict discharge disposition,,$^{20}$ and, as most neurosurgical patients require coordinated transport home, the predetermined discharge time allowed the TCP team to prearrange means for departure, further reducing patients' hospital wait time.

Of note, the intensive efforts of the TCP facilitated morning discharge for $40 \%$ of patients, but obstacles to a greater proportion of morning discharges are worth discussing. A major structural impediment was our discharge system protocol. At our institution, doctors and nurses perform separate discharge reports, and, historically, the nursing discharge was performed after the physician's on the day of discharge, hindering file completion within the allotted time frame. Since conducting this analysis, we have changed our system so that nursing staff performs their discharge the evening prior to the actual discharge date, and we believe that this change will improve our rate of discharges before noon. There were also limitations in the process for discharge order entry; the patient was not considered formally discharged until an off-floor unit coordinator recorded the discharge time. Discordance between true discharge and documented discharge has been reconciled with the implementation of the electronic medical record.

The implicit learning curve during system implementation was another obstacle. In this large academic setting, the house staff and fellows frequently rotate between services, and, although new team members were educated about the fundamentals of the program, expectations of discharge timing were not always conveyed. This has improved with continued teaching and institutional awareness of the program. The attendance of discharge appointments by patients' family members was also a limitation. Caregivers were often delayed, which postponed discharge education and occasionally resulted in appointment cancellations. Going forward, we plan to place additional emphasis on the importance of prompt arrival to the discharge meeting to patients, caregivers, and hospital staff.

In striving for efficiency of care, particularly regarding shorter hospitalizations, one concern is a theoretical resul- 
tant increase in preventable readmissions, ${ }^{12}$ as was seen in a neurosurgical short-stay protocol that was applied to reduce hospital admissions for lumbar discectomy. ${ }^{29}$ In that case, the outpatient protocol allowed for $49.7 \%$ of patients to go home the day of surgery, compared with only $3.6 \%$ having same-day discharges before, although readmission rates did increase (from $2.3 \%$ to $4.6 \%$ ). However, this was not seen in the current study, as the TCP was associated with significantly decreased odds of unplanned readmission. Questions surrounding adverse consequences of shorter hospitalizations may be addressed with a combination of patient (and family) education with postdischarge surveillance. 2,38 In this intervention, patient education greatly enhanced patient understanding of normal versus abnormal postoperative events. The use of structured and culturally sensitive education was critical to the success of the program, and these findings echo those of other transitional care systems that have described the fundamental importance of patient education. ${ }^{17,26}$

Multiple reports have demonstrated that medication adherence is important for readmission prevention. ${ }^{14,18,21}$, $28,40,41,43$ The experience of participating surgeons was that noncompliance with medication regimens, potentially due to inadequate education, was a driver of readmission on the neurosurgical service. Therefore, a list of important medications was identified and delivered to patients prior to discharge, which may have lessened avoidable readmissions because of noncompliance. Postoperative education was followed by postdischarge telephone contact, which identified any remaining questions. In the patient surveys, greater than $97 \%$ of TCP participants reported a strong understanding of their role in postoperative care (medication regimen, incision care, nutrition, and physical activity), and how to follow up postoperatively, even in case of an emergency. Patients who were not TCP enrollees reported poor understanding of these objectives. These results align with previous reports that pharmacy assistance and education, as well as posthospitalization surveillance, aid in reducing avoidable readmissions. ${ }^{14,18,21,28,40,41,43}$

The quality improvement seen with the TCP was multifaceted. The team included educational consultants, digital media experts, nurses, pharmacists, physician assistants, physicians, and hospital administrators. The importance of patient education and engagement has already been well described in the medical literature, ${ }^{15,27}$ and educationintensive, surgical discharge appointments led to higher quality care in this study. The TCP was also associated with high patient satisfaction. Proactive management of patients' expectations surrounding discharge, implementation of the transitional care bundle, and providing patients with a specific discharge appointment when scheduling surgery facilitated patients' understanding of their overall hospital course and the transition to home process.

This study has a number of limitations. Although the sample size was relatively large, the TCP only included a subset of elective neurosurgical cases, and generalizability of results when scaling a TCP to an entire department or institution awaits confirmation. Generalizability to other institutions is also unknown. Additionally, readmission results only capture those reentering the same hospital, which would underestimate the overall readmission rate if patients were admitted to other hospitals. Finally, future research on the cost-effectiveness of this program is needed. We are presently performing an analysis of costs and benefits of the program. This incorporates hospital expenditures for both personnel and in-house prescription filling for essential medications, as well as the cost of educational materials, contrasted with benefits of hospital bed vacancy opportunity costs, and the amount of money saved by the health care system with reduced readmissions. Patient satisfaction is another critical component of value, and, while we reported the results of voluntary surveys, there was a small number of respondents from the non-TCP group. We plan to acquire institutional Press Ganey data to better understand the difference in satisfaction between the 2 groups.

Overall, targeted enhancement of transitional care improved neurosurgical patients' safety and experience while simultaneously increasing hospital bed availability and improving patient progression. Our results highlight the utility of a comprehensive transitional care program for hospital discharge surrounding surgical care among similar fields and similar hospitals. It is our plan to continue to expand the tools used in this transitional care program to include risk stratification to guide insurance preapproval for additional services, formalized group classes, and increased use of technology to monitor patients after discharge.

\section{Conclusions}

The transitional care program is a novel approach designed to improve the postoperative care after elective neurosurgery, and included predetermined anticipated discharge dates, enhanced education at patient discharge, and postdischarge surveillance. This multidisciplinary effort reduced the length of hospitalization, promoted early discharge, and reduced readmissions while enhancing the patient experience and quality of care.

\section{References}

1. Baker DW, Gazmararian JA, Williams MV, Scott T, Parker RM, Green D, et al: Functional health literacy and the risk of hospital admission among Medicare managed care enrollees. Am J Public Health 92:1278-1283, 2002

2. Basques BA, Varthi AG, Golinvaux NS, Bohl DD, Grauer JN: Patient characteristics associated with increased postoperative length of stay and readmission after elective laminectomy for lumbar spinal stenosis. Spine (Phila Pa 1976) 39:833-840, 2014

3. Bates OL, O'Connor N, Dunn D, Hasenau SM: Applying STAAR interventions in incremental bundles: improving post-CABG surgical patient care. Worldviews Evid Based Nurs 11:89-97, 2014

4. Berkowitz RE, Fang Z, Helfand BK, Jones RN, Schreiber R, Paasche-Orlow MK: Project ReEngineered Discharge (RED) lowers hospital readmissions of patients discharged from a skilled nursing facility. J Am Med Dir Assoc 14:736-740, 2013

5. Bernatz JT, Anderson PA: Thirty-day readmission rates in spine surgery: systematic review and meta-analysis. Neurosurg Focus 39(4):E7, 2015

6. Bina RW, Lemole GM, Dumont TM: Measuring quality of neurosurgical care: readmission is affected by patient factors. World Neurosurg 88:21-24, 2016 
7. Brown EG, Bold RJ: Hospital readmissions: are they preventable? Adv Surg 49:15-29, 2015

8. Ceppa EP, Pitt HA, Nakeeb A, Schmidt CM, Zyromski NJ, House MG, et al: Reducing readmissions after pancreatectomy: limiting complications and coordinating the care continuum. J Am Coll Surg 221:708-716, 2015

9. Charlson ME, Pompei P, Ales KL, MacKenzie CR: A new method of classifying prognostic comorbidity in longitudinal studies: development and validation. J Chronic Dis 40:373383, 1987

10. Clari M, Frigerio S, Ricceri F, Pici A, Alvaro R, Dimonte V: Follow-up telephone calls to patients discharged after undergoing orthopaedic surgery: double-blind, randomised controlled trial of efficacy. J Clin Nurs 24:2736-2744, 2015

11. CMS: Readmissions reduction program. CMS.gov (https:// www.cms.gov/medicare/medicare-fee-for-service-payment/ acuteinpatientpps/readmissions-reduction-program.html) [Accessed March 24, 2017]

12. Dasenbrock HH, Liu KX, Devine CA, Chavakula V, Smith TR, Gormley WB, et al: Length of hospital stay after craniotomy for tumor: a National Surgical Quality Improvement Program analysis. Neurosurg Focus 39(6):E12, 2015

13. Delisle DR: Big things come in bundled packages: implications of bundled payment systems in health care reimbursement reform. Am J Med Qual 28:339-344, 2013

14. Dharmarajan K, Hsieh AF, Lin Z, Bueno H, Ross JS, Horwitz LI, et al: Hospital readmission performance and patterns of readmission: retrospective cohort study of Medicare admissions. BMJ 347:f6571, 2013

15. Etkind SN, Daveson BA, Kwok W, Witt J, Bausewein C, Higginson IJ, et al: Capture, transfer, and feedback of patientcentered outcomes data in palliative care populations: does it make a difference? A systematic review. J Pain Symptom Manage 49:611-624, 2015

16. Flannigan RK, Gotto GT, Donnelly B, Carlson KV: Standardized follow-up program may reduce emergency room and urgent care visits for patients undergoing radical prostatectomy. Can Urol Assoc J 8:E505-E509, 2014

17. Frank-Bader M, Beltran K, Dojlidko D: Improving transplant discharge education using a structured teaching approach. Prog Transplant 21:332-339, 2011

18. Gilmore V, Efird L, Fu D, LeBlanc Y, Nesbit T, Swarthout M: Implementation of transitions-of-care services through acute care and outpatient pharmacy collaboration. Am J Health Syst Pharm 72:737-744, 2015

19. Godil SS, Parker SL, Zuckerman SL, Mendenhall SK, Devin CJ, Asher AL, et al: Determining the quality and effectiveness of surgical spine care: patient satisfaction is not a valid proxy. Spine J 13:1006-1012, 2013

20. Halawi MJ, Vovos TJ, Green CL, Wellman SS, Attarian DE, Bolognesi MP: Patient expectation is the most important predictor of discharge destination after primary total joint arthroplasty. J Arthroplasty 30:539-542, 2015

21. Hansen LO, Young RS, Hinami K, Leung A, Williams MV: Interventions to reduce 30-day rehospitalization: a systematic review. Ann Intern Med 155:520-528, 2011

22. Jack BW, Chetty VK, Anthony D, Greenwald JL, Sanchez GM, Johnson AE, et al: A reengineered hospital discharge program to decrease rehospitalization: a randomized trial. Ann Intern Med 150:178-187, 2009

23. Jencks SF, Williams MV, Coleman EA: Rehospitalizations among patients in the Medicare fee-for-service program. $\mathbf{N}$ Engl J Med 360:1418-1428, 2009

24. Joynt KE, Orav EJ, Jha AK: Thirty-day readmission rates for Medicare beneficiaries by race and site of care. JAMA 305:675-681, 2011

25. Kalogianni A, Almpani P, Vastardis L, Baltopoulos G, Charitos C, Brokalaki H: Can nurse-led preoperative education reduce anxiety and postoperative complications of pa- tients undergoing cardiac surgery? Eur J Cardiovasc Nurs 15:447-458, 2016

26. Katz JN, Martin TL: Clinical pathways and the commodification of total joint replacement. Osteoarthritis Cartilage 20:1057-1058, 2012

27. Koelling TM, Johnson ML, Cody RJ, Aaronson KD: Discharge education improves clinical outcomes in patients with chronic heart failure. Circulation 111:179-185, 2005

28. Krumholz HM, Amatruda J, Smith GL, Mattera JA, Roumanis SA, Radford MJ, et al: Randomized trial of an education and support intervention to prevent readmission of patients with heart failure. J Am Coll Cardiol 39:83-89, 2002

29. Lang SS, Chen HI, Koch MJ, Kurash L, McGill-Armento KR, Palella JM, et al: Development of an outpatient protocol for lumbar discectomy: our institutional experience. World Neurosurg 82:897-901, 2014

30. Lepänluoma M, Rahi M, Takala R, Löyttyniemi E, Ikonen TS: Analysis of neurosurgical reoperations: use of a surgical checklist and reduction of infection-related and preventable complication-related reoperations. J Neurosurg 123:145152, 2015

31. Louis-Simonet M, Kossovsky MP, Sarasin FP, Chopard P, Gabriel V, Perneger TV, et al: Effects of a structured patientcentered discharge interview on patients' knowledge about their medications. Am J Med 117:563-568, 2004

32. Maniaci MJ, Heckman MG, Dawson NL: Functional health literacy and understanding of medications at discharge. Mayo Clin Proc 83:554-558, 2008

33. Marcus LP, McCutcheon BA, Noorbakhsh A, Parina RP, Gonda DD, Chen C, et al: Incidence and predictors of 30-day readmission for patients discharged home after craniotomy for malignant supratentorial tumors in California (19952010). J Neurosurg 120:1201-1211, 2014

34. Moghavem N, Morrison D, Ratliff JK, Hernandez-Boussard T: Cranial neurosurgical 30-day readmissions by clinical indication. J Neurosurg 123:189-197, 2015

35. Naylor MD, Brooten DA, Campbell RL, Maislin G, McCauley KM, Schwartz JS: Transitional care of older adults hospitalized with heart failure: a randomized, controlled trial. J Am Geriatr Soc 52:675-684, 2004

36. Porter ME: What is value in health care? N Engl J Med 363:2477-2481, 2010

37. Rambachan A, Smith TR, Saha S, Eskandari MK, Bendok BR, Kim JY: Reasons for readmission after carotid endarterectomy. World Neurosurg 82:e771-e776, 2014

38. Reponen E, Korja M, Niemi T, Silvasti-Lundell M, Hernesniemi J, Tuominen H: Preoperative identification of neurosurgery patients with a high risk of in-hospital complications: a prospective cohort of 418 consecutive elective craniotomy patients. J Neurosurg 123:594-604, 2015

39. Sanchez GM, Douglass MA, Mancuso MA: Revisiting Project Re-Engineered Discharge (RED): The impact of a pharmacist telephone intervention on hospital readmission rates. Pharmacotherapy 35:805-812, 2015

40. Sands DZ, Safran C: Closing the loop of patient care-a clinical trial of a computerized discharge medication program. Proc Annu Symp Comput Appl Med Care 1994:841-845, 1994

41. Scott IA: Preventing the rebound: improving care transition in hospital discharge processes. Aust Health Rev 34:445451, 2010

42. Shah MN, Stoev IT, Sanford DE, Gao F, Santiago P, Jaques DP, et al: Are readmission rates on a neurosurgical service indicators of quality of care? J Neurosurg 119:1043-1049, 2013

43. Stevens S: Preventing 30-day readmissions. Nurs Clin North Am 50:123-137, 2015

44. Szöts K, Konradsen H, Solgaard S, Bogø S, Østergaard B: Nurse-led telephone follow-up after total knee arthroplasty- 
content and the patients' views. J Clin Nurs 24:2890-2899, 2015

45. Thomas JG, Gadgil N, Samson SL, Takashima M, Yoshor D: Prospective trial of a short hospital stay protocol after endoscopic endonasal pituitary adenoma surgery. World Neurosurg 81:576-583, 2014

46. Tsai TC, Joynt KE, Orav EJ, Gawande AA, Jha AK: Variation in surgical-readmission rates and quality of hospital care. N Engl J Med 369:1134-1142, 2013

47. Tummers JF, Schrijvers AJ, Visser-Meily JM: Economic evidence on integrated care for stroke patients; a systematic review. Int J Integr Care 12: e193, 2012

48. Vaziri S, Cox JB, Friedman WA: Readmissions in neurosurgery: a qualitative inquiry. World Neurosurg 82:376-379, 2014

49. Vest JR, Gamm LD, Oxford BA, Gonzalez MI, Slawson KM: Determinants of preventable readmissions in the United States: a systematic review. Implement Sci 5:88, 2010

50. Williams MV, Li J, Hansen LO, Forth V, Budnitz T, Greenwald JL, et al: Project BOOST implementation: lessons learned. South Med J 107:455-465, 2014

\section{Disclosures}

The authors report no conflict of interest concerning the materials or methods used in this study or the findings specified in this paper.

\section{Author Contributions}

Conception and design: Gormley, Logsdon, Raftery. Acquisition of data: Gormley, Robertson, Logsdon, Dasenbrock, Raftery. Analysis and interpretation of data: Gormley, Robertson, Dasenbrock, Yan, Raftery, Smith. Drafting the article: Robertson, Dasenbrock. Critically revising the article: Gormley, Robertson, Logsdon, Dasenbrock, Smith. Reviewed submitted version of manuscript: all authors. Approved the final version of the manuscript on behalf of all authors: Gormley. Statistical analysis: Robertson, Dasenbrock, Yan, Smith. Administrative/technical/ material support: Gormley, Logsdon, Raftery. Study supervision: Gormley, Logsdon, Raftery.

\section{Supplemental Information}

\section{Online-Only Content}

Supplemental material is available with the online version of the article.

Supplemental Table 1. https://thejns.org/doi/suppl/10.3171/ 2017.2.JNS161770

\section{Correspondence}

William B. Gormley, Department of Neurological Surgery, Brigham and Women's Hospital and Harvard Medical School, 75 Francis St., Boston, MA 02115. email: wgormley@partners.org. 